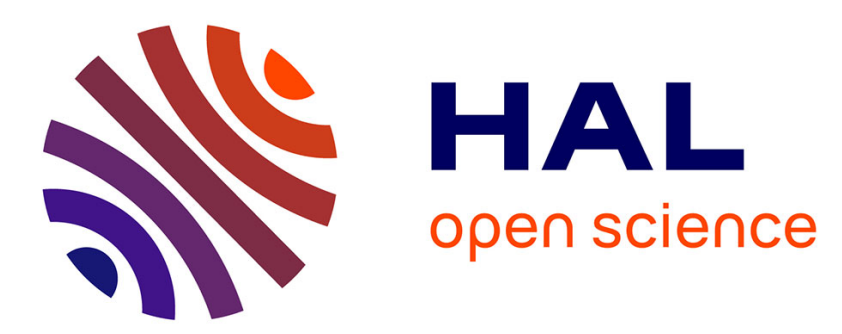

\title{
Stability and Transparency Analysis of a Teleoperation Chain For Microscale Interaction.
}

\author{
A. Mohand Ousaid, Aude Bolopion, Sinan Haliyo, Stéphane Régnier, Vincent
} Hayward

\section{- To cite this version:}

A. Mohand Ousaid, Aude Bolopion, Sinan Haliyo, Stéphane Régnier, Vincent Hayward. Stability and Transparency Analysis of a Teleoperation Chain For Microscale Interaction.. 2014 IEEE International Conference on Robotics and Automation (ICRA 2014), May 2014, Hong Kong, China. pp.5946-5951, 10.1109/ICRA.2014.6907735 . hal-01313511

\section{HAL Id: hal-01313511 \\ https://hal.science/hal-01313511}

Submitted on 10 May 2016

HAL is a multi-disciplinary open access archive for the deposit and dissemination of scientific research documents, whether they are published or not. The documents may come from teaching and research institutions in France or abroad, or from public or private research centers.
L'archive ouverte pluridisciplinaire HAL, est destinée au dépôt et à la diffusion de documents scientifiques de niveau recherche, publiés ou non, émanant des établissements d'enseignement et de recherche français ou étrangers, des laboratoires publics ou privés. 


\title{
Stability and Transparency Analysis of a Teleoperation Chain For Microscale Interaction
}

\author{
A. Mohand Ousaid*, A. Bolopion ${ }^{+}$, S. Haliyo*, S. Régnier* and V. Hayward*
}

\begin{abstract}
Microscale teleoperation with haptic feedback requires scaling gains in the order of $10^{4}-10^{7}$. These high gains impose a trade-off between stability and transparency. Due to the conservative approach used in most designs, transparency is reduced since damping is added to the system to guarantee stability. Starting from the fact that series, negative feedback and parallel connection of passive systems is a passive system, a new approach is addressed in this work. We propose here a complete teleoperation chain designed from the ground up for full transparency and stability, including a novel self-sensing probe and a high fidelity force-feedback haptic interface. By guaranteeing the passivity of each device and assuming that the human operator and the environment are passive systems, a homothetic direct coupling can be used without jeopardizing the stability and provides best transparency. The system is experimentally demonstrated in the complex case of a probe interacting with a water droplet under human control, while accurately transcribing the interaction back to operator.
\end{abstract}

Index Terms-Bilateral control, microscale teleoperation, passivity, transparency, stability.

\section{INTRODUCTION}

Human interaction with the microscale remains an important challenge for several applications in nanotechnology, biology and physics such as characterization and analysis of microscale phenomena. In fact, at this scale the operators cannot interact directly with objects because of the scale and especially of severe differences in physics: mechanical behavior is no longer dictated by gravity, and short range forces such as electrostatic, capillary and van der Waals forces dominate [1]. Bilateral micro teleoperation in this case appears to be an interesting solution to supplement human perception and assist the operator in his/her task [2], [3]. Two issues arise in this case: stability and transparency. The first is a necessary condition for the usability of the system, while the second relates to the ability of the setup to provide the operator with an accurate feeling of the microscale interaction [4], [5]. Since 1990, several micro teleoperation systems have been proposed to provide a sense of touch and to enhance manipulation capabilities at the microscale. As an early example, Hamatura and Morishita proposed a nanomanipulation system to allow human operators to experience the "nanometer world" [3]. Hollis introduced haptic feedback for the first time using a Scanning

\footnotetext{
* are with Institut des Systèmes Intelligents et de Robotique, Université Pierre et Marie Curie, CNRS UMR 7222, 4 Place Jussieu, 75252 Paris Cedex, France \{mohand_ ousaid, haliyo, regnier, hayward\}@isir.upmc.fr

+ is with AS2M department - FEMTO-ST Institute, CNRS UMR 6174 UFC, ENSMM, UTBM aude.bolopionefemto-st.fr
}

Tunneling Microscope (STM) [2]. Lawrence proposed tools for quantifying performances of general teleoperation system architecture, stability and transparency [4]. Following those works, Sitti and Hashimoto connected an haptic interface with an Atomic Force Microscope (AFM) to feel and manipulate small objects, using homothetic gains to adapt micro and macro scales [6]. Ryu and Hannaford also proposed a control scheme to ensure stable teleoperation under a wide variety of environments and operating speeds based on passivity control [7]. In [8], a bilateral controller based on [9] was presented using passivity approaches to deal with time delay for microscale operation. In recent literature, various approaches (passivity, oblique coordinates, observers...) are used to improve performances of bilateral micro teleoperation systems, especially in terms of stability [10]-[18]

However, no work achieved in the same time stability and transparency of the micro teleoperation systems. All of the above-mentioned systems are subjected to the classical engineering trade-off between stability and transparency. Due to the conservative approaches used in those designs, transparency is reduced since damping is added to the system to guarantee stability. On the other hand, a micro to macro teleoperation system requires homothetic gains in the order of $10^{4}$ to $10^{7}$. Those scaling factors greatly fragilize stability, especially in the presence of uncertainties like disturbances or sensor noise. Recently, a study shows that unlike indirect coupling, the direct coupling (Direct Force Feedback control scheme) that uses only homothetic gains is transparent but it suffers from a trade-off between force amplification and workspace limitation [19]. Furthermore, transparency is generally affected by the mechanical properties of the haptic interface. For example, small forces are badly transmitted due to problems of friction, inertia and hysteresis that tend to deteriorate the feeling provided by such systems

To overcome those limitations and this trade-off, we propose in this paper a new approach. Instead of using available devices with a dedicated bilateral control scheme to ensure the overall stability, we propose a modular micro teleoperation system using custom components designed with passivity and transparency constraints. The stability is then guaranteed as the serial connection of passive systems yield a passive system [20]. The used haptic interface is presented in [21]. Its design provides performances covering human haptic perception and avoids the mechanical degradation of the feedback signal. Its transparency is shown on a large bandwidth [22]. The microscale probe and the associated force sensor are described in detail in [23]. A direct coupling 
scheme is proposed here to provide a stable and transparent microscale teleoperation chain using these components. This connection of passive components overcomes the need to sacrifice the transparency for stability. Tools based on passivity and impedance analysis are introduced to evaluate the stability and the transparency. An experimental case study is presented, where a human operator probes a water droplet. The haptic feedback is a faithful replica of the microscale interaction, due to the accuracy of the force sensor and the haptic interface, and the used direct coupling scheme.

This paper is composed of six sections. Passivity theory is addressed in section II. In section III and IV, the master system and slave system used in this work are presented respectively. The stability and transparency of the bilateral teleoperation system are analysed and the validity of the proposed approach is confirmed by experiments in section $\mathrm{V}$. In the last section, the work is summarized.

\section{PASSIVITY THEORY}

Theoretical proof of the stability of any interconnected system is quite difficult, if not impossible, to provide. The passivity concept has consequently attracted great interests because it gives an easier way to prove the stability [24].

Passivity is defined such that a given system with state $x$, input $u$ and output $y$ is said passive if there exists a storage function $V(x) \geq 0$, with an initial stored energy $V(0)$, and a real-valued rate $W(u, y)$, such that the inequality (1) holds $\forall t \geq 0$.

In other words, the stored energy of the system is always less than or equal to the sum of the initial stored energy and the energy provided to the system. A passive system is internally stable [25]-[27].

$$
(V(x(t))-V(x(0))) \leqslant \int_{0}^{t} W(u(\tau), y(\tau)) \mathrm{d} \tau
$$

The passivity concept provides a strong tool to study the stability of systems and interconnected systems [28]. Any serial, parallel or negative feedback combination of two passive subsystems is also passive [20], [29]. According to the Parseval's Theorem [30], [31] the passivity of a LTI (Linear Time Invariant) system with a rational transfer function $H$ is guaranteed if

(i) for the continuous time case

- all poles of $H(s)$ have real parts less than zero;

- and $H(j w)+H^{*}(j w) \geqslant 0$ for all $w \in[-\infty \infty]$

(ii) for the discrete time case

- all poles of $H(z)$ have a module less than one;

- and $H\left(e^{j \theta}\right)+H^{*}\left(e^{-j \theta}\right) \geqslant 0$ for all $\theta \in\left[\begin{array}{ll}0 & 2 \pi\end{array}\right]$

where the superscript ${ }^{*}$ denotes complex conjugate transposition. If a LTI system is passive, the system is stable when coupled to an arbitrary passive system which is itself passive [32].

These criteria are used to verify the passivity of slave and master devices. In this work no distinction is made between the continuous and the discrete case, since the sampling

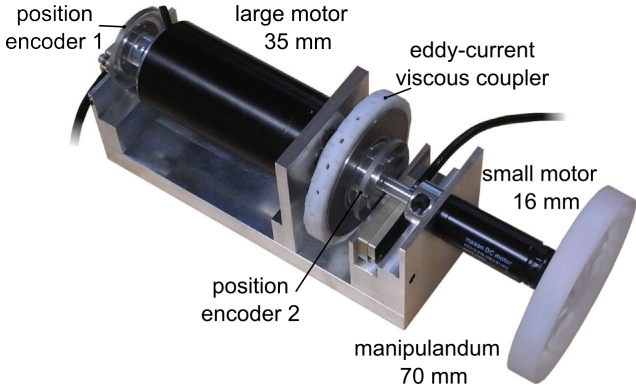

Fig. 1. Dual stage haptic interface. A large and small motor are coupled through a magnetic passive clutch based on eddy currents. The output torque is a linear function of the differential velocity of both motors summed to the torque provided by the small motor.

frequency is ten times higher than the bandwidth of the system $\left(f_{e}=\frac{1}{T_{e}}=1 \mathrm{kHz}\right)$.

\section{MASTER SYSTEM: DUAL STAGE HAPTIC INTERFACE}

The haptic interface with one degree of freedom [21] used in this work is shown in Fig. 1. Haptic interfaces using electromagnetic drives are subjected to an inherent inertiatorque trade-off which limits transparency : the higher the torque, the higher the inertia. Consequently the transparency is degraded with inertia.

The proposed interface is based on a dual-stage architecture. It is comprised of a large motor coupled to a small one, also carrying the handle, through a passive viscous clutch based on eddy currents. This clutch mechanism accurately transforms slip velocity of the large motor into torque and exhibits a linear behavior on a large bandwidth (for brevity, no distinction is made in the following between forces and torques). This first order relationship between the velocity control and the handle eliminates the dynamics of the large motor from the user experience. A feed-forward path is provided through the small motor to fill in the transients or fast variations of the input signal that the large motor could not provide. As this second motor has negligible friction and inertia, unwanted forces could be kept below human detection threshold achieving quasi-perfect transparency.

\section{A. Haptic interface control scheme}

Referring to Fig. 2, the system has two torque commands and one torque output, which can potentially provide a number of control options. Here, the primary control objective is regulation and tracking of the output torque $\tau_{\text {out }}$.

The reference torque $\tau_{d}$, is compared to the torque produced by the clutch, $\tau_{\text {coupler }}$, which is proportional to the relative velocity between the large and small motors. The large motor is enslaved by the compensator $C(z)$ to the torque error. The small motor is controlled to reduce the error without compensation since it is needed to transiently compensate for the slower response of the large motor. If 
the regulation is good enough, when the handle is backdriven, the power required to move the large motor must be entirely supplied by the power amplifier, not by the user. A polynomial controller is used to achieve this objective.

Figure 3 represents the response of the interface to a null reference. The controller compensates the inertia of the large motor. The dynamic range of the interface covers from $5.2 \mathrm{mN}$ to $5.7 \mathrm{~N}$. Furthermore, the control scheme doesn't change the passivity of the haptic interface according to the properties of passive interconnected systems described in section II.

\section{B. Interface performances}

User tests were carried out to evaluate the performances of the interface in [22]. The aim of the experiment was to validate the high degree of transparency of the device. The operator had to perform a task of detecting a small amplitude stimulus, which can be assimilated to scanning a planar surface at the microscale with a localized minor defect. The result of the tests shows that the interface achieves one order of magnitude of improvement in transparency over existing designs, i.e ten times smaller stimuli can be accurately detected. The inertial load seen by a finger is only around one gram.

\section{SlaVE SySTEM : ACTIVE MICRO FORCE SENSOR}

The slave device performs the physical interaction at the microscale and provides force sensing. The force sensor is presented in depth in [23]. The sensor uses electrostatic energy instead of elastic energy to perform the measurement. It is designed around a linear comb-drive actuator. Its working principle is to keep the probe on its equilibrium position by a force to cancel out external forces. As the comb-drive actuator is perfectly known and controlled, the balancing force is equal to the external force and directly provides the measure (Fig. 4).

Based on the position feedback of the probe $y(t)$ an $\mathrm{H}$ infinity controller generates an opposite signal $u(t)$ in order to balance the external applied force and to maintain the probe at its equilibrium position, as shown in Fig. 5.

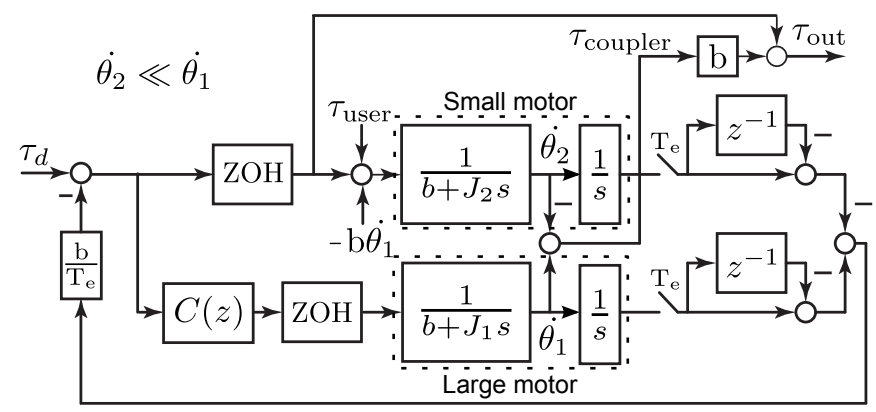

Fig. 2. Torque control scheme of the haptic interface.

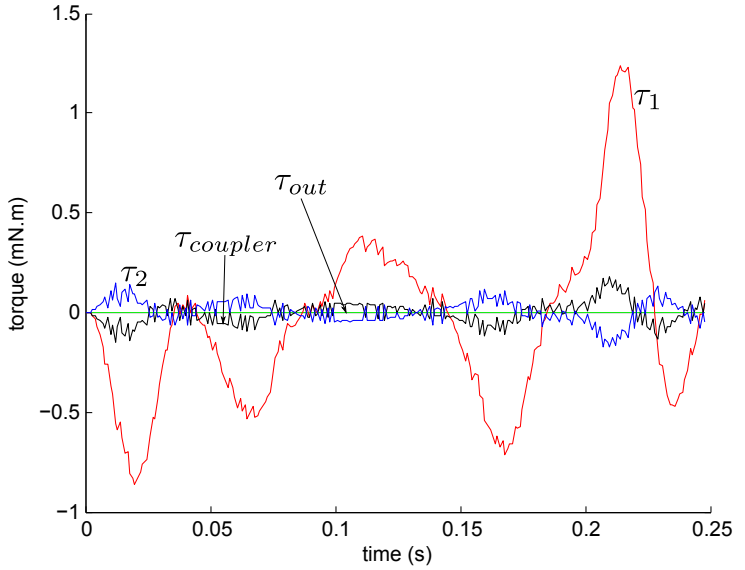

Fig. 3. Haptic interface response to a null reference. As the controller compensates the inertia of the large motor, the torque felt by the user is null.

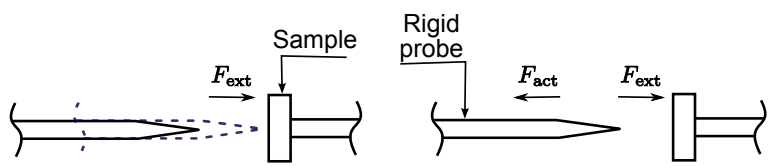

(a) Before balance

(b) After balance

Fig. 4. Force balance or compensation principle. (a) The sensor probe is attracted by the sample with a force $F_{e x t}$, while (b) the controller maintains the probe at the equilibrium position by applying a balance force $F_{\text {act }}$ to compensate the external force.

Instead of measuring the interaction using the signal coming from the displacement of the probe, it is computed as being proportional to the value of the opposite voltage applied by the controller generating the opposite force $F_{a c t}$. In this case, $F_{\text {act }} \simeq-F_{\text {ext }}$. This technique which uses the closedloop control principle allows maintaining the sensitive part of the sensor in the linear range, improving precision, increasing the sensor dynamic range and improving the robustness and the stability of the sensor. Thus, the sensor probe can track the integrality of the tip-sample interaction curves. It especially avoids several problems inherent to scanning probe microscopy using elastic probes, such as pull-in, pull-off and hysteresis.

The LTI transfer function $H(s)$ linking $F_{\text {act }}$ to $F_{\text {ext }}$ is:

$$
H(s)=\frac{G(s) K(s)}{1+G(s) K(s)}
$$

where $G(s)$ and $K(s)$ are respectively the system plant and

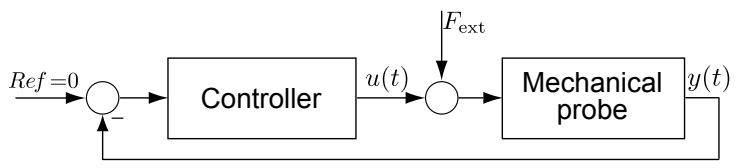

Fig. 5. Active force sensing control scheme. The controller keeps the probe at its equilibrium position in order to balance external force $F_{\text {ext }}$. This principle provides directly the measure of the force. 


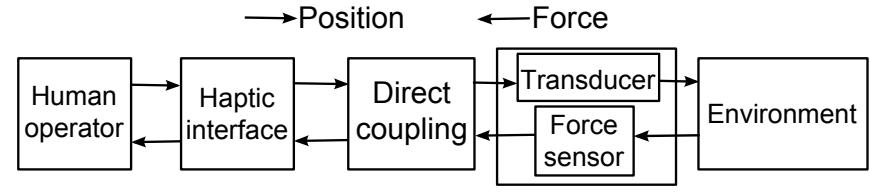

Fig. 6. Series system blocks connection. Blocks are (left to right) : operator, haptic interface, coupling, sensor and transducer, environment.

the $\mathrm{H}_{\infty}$ controller [23]:

$$
G(s)=\frac{k_{n} w_{n}^{2}}{s^{2}+2 \zeta w_{n} s+w_{n}^{2}}
$$

where $k_{n}=0.032, \zeta=0.0047$ and $w_{n}=64 \mathrm{rad} / \mathrm{s}$ and

$$
K(s)=\frac{78.4 s^{3}+1.56810^{8} s^{2}+7.46810^{7} s+3.86110^{11}}{s^{4}+7516 s^{3}+2.57110^{6} s^{2}+3.12410^{8} s+1.09910^{8}}
$$

All the poles of the function $H(s)$ have a negative real part and the real part of the transfer function is positive over the entire bandwidth. This verifies the LTI passivity statements given above. The system thus remains passive over the entire bandwidth.

\section{BILATERAL MICRO TELEOPERATION SYSTEM}

A direct force feedback control scheme is used for the bilateral coupling. This scheme is to be the most intuitive formulation to accurately provide amplified forces to the operator. It is achieved by two homothetic gains $\alpha_{f}$ and $\alpha_{d}$, respectively the force and the position scaling factors. These gains are necessary to adjust the scale between the macro and micro environment. As this coupling is solely comprised of those linear gains, the transparency is ideal, as shown in [33]. The gain $\alpha_{f}$ is chosen according to the dynamic range of the sensor and of the haptic interface. Its value in this case is $0.0510^{6}$. The gain $\alpha_{d}$ is chosen according to the displacement range of the sample holder and the handle. For a good trade-off between precision and manipulation comfort, the value of $1 / \alpha_{d}=14010^{-4}$ is used.

As shown in Fig. 8, the position of the handle is scaled down and used as position set point to the transducer carrying the sample holder. Its transfer function $M_{p}(s)\left(M_{p}(z)\right)$ can be considered as unity, $M_{p}(s)=M_{p}(z) \simeq 1$. The force measured by the sensor is scaled up as force set point to the haptic interface.

\section{A. Stability analysis}

The whole system can be presented as a series connection of sub-systems (blocks) as depicted in Fig. 6. The system is composed of the haptic interface connected to the human operator and to the slave device (considered as the sum of the transducer of the sample holder and the force sensor) through direct haptic coupling. The slave device is connected to the environment.

Assuming that the user will not voluntarily destabilize the system, his/her hand is considered as a passive system [34].

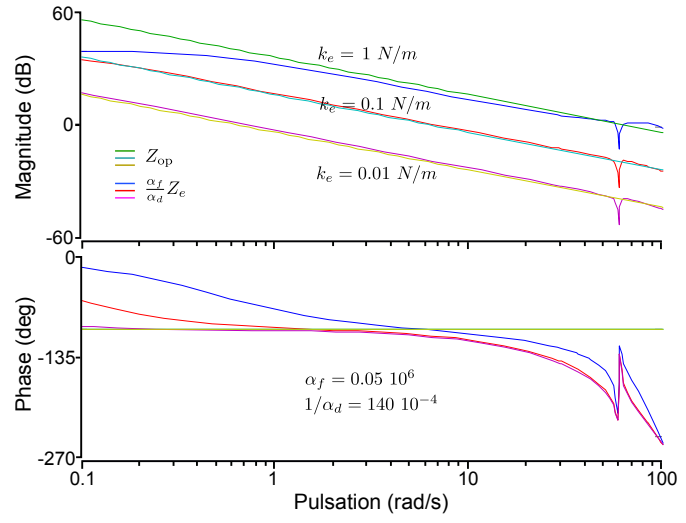

Fig. 7. Impedance bode diagram, contact mode $\left(k_{e}=0.01,0.1\right.$ and $\left.1 \mathrm{~N} / \mathrm{m}\right)$.

The environment is also assumed to be passive as there is no time delay. As the direct coupling itself is passive, the passivity of the whole system is then guaranteed since the series connection of passive systems yield a passive system as detailed previously. As shown in [32], if a LTI system is passive, the system is stable when coupled to arbitrary passive system which is itself passive. This provides a sufficient condition for the stability of the micro teleoperation system presented in this paper.

\section{B. Transparency analysis}

To analyze the transparency of the system, the impedance criterion is used in this paper. The operator side impedance $Z_{o p}=\frac{F_{o p}}{X_{h}}$ is compared with the environment side impedance $Z_{e}=\frac{F_{e}}{X_{e}}$. The scaling gains are also taken into account. The perfect transparency is achieved [4] if :

$$
Z_{o p}=\frac{\alpha_{f}}{\alpha_{d}} Z_{e}
$$

The transparency investigation is carried out for two specific cases: a slave in non-contact mode $\left(Z_{e} \rightarrow 0\right.$, no applied force) and a slave in contact mode. For the first mode (non-contact mode), the operator-side impedance depends only on the characteristics of the haptic interface's small motor. As shown in [21], the choice of this motor is made to ensure minimal inertia. Thus, the ideal transparency is achieved for this mode. For the second mode (contact mode), the environment is modeled as a sample with a stiffness $k_{e}$. Figure 7 represents a Bode diagram of $Z_{o p}$ and of $\frac{\alpha_{f}}{\alpha_{d}} Z_{e}$ for three values of $k_{e}$ representing typical stiffness values of soft matter. The result shows that the impedance of the micro environment is transmitted to the human operators for $k_{e}<1 \mathrm{~N} / \mathrm{m}$. The bandwidth of the sensor currently limits the stiffness of the samples that can be sensed up to $1 \mathrm{~N} / \mathrm{m}$. By increasing the sensor bandwidth, the system could interact with stiffer samples.

\section{System validation}

1) Experimental bench: The experimental setup is depicted in Fig. 8(b). The sensor is placed on an anti-vibration 


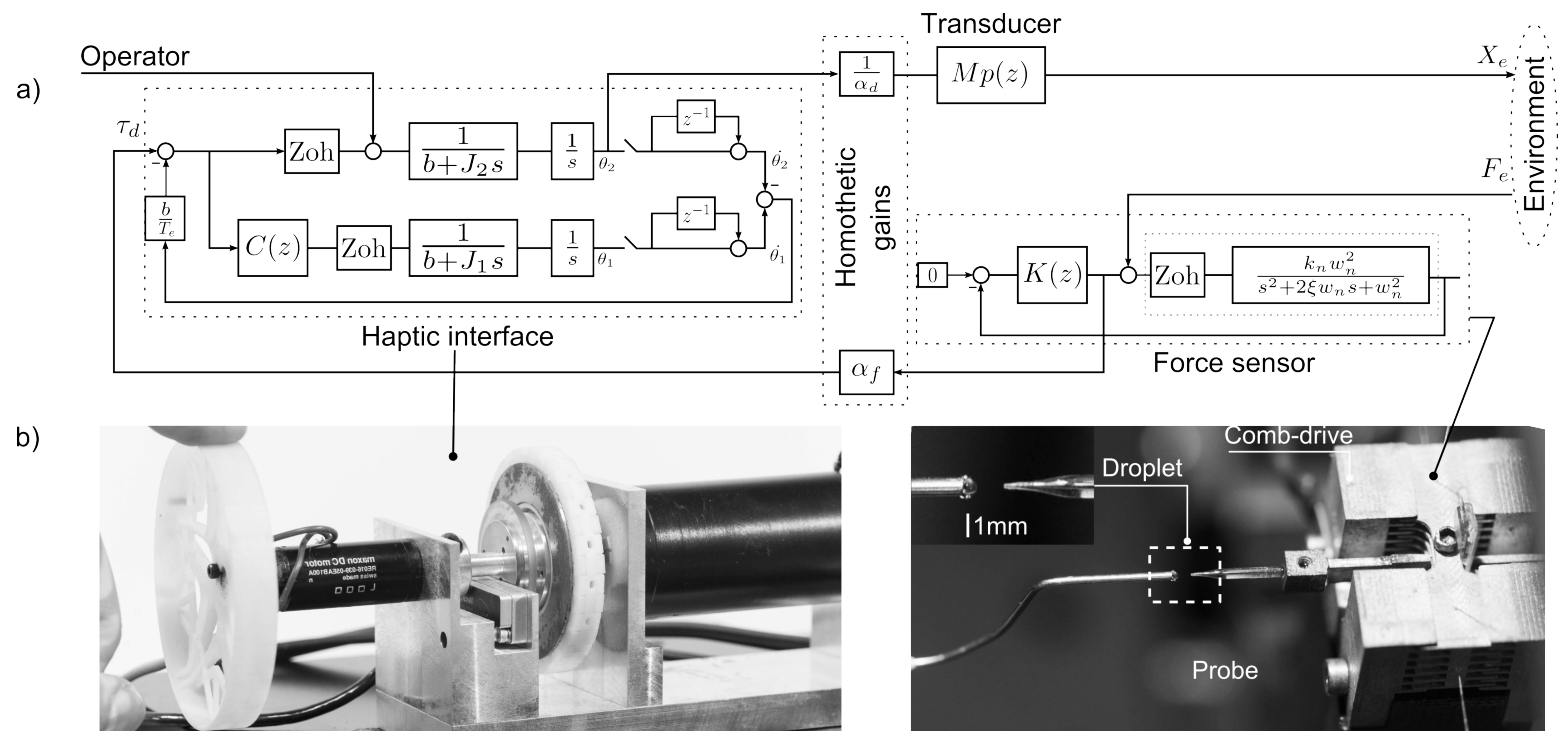

Fig. 8. Global view of the micro teleoperation system. (a) System architecture, (b) Experimental setup.

table to avoid external mechanical perturbations. An optical microscope provides a visual feedback of the sample (droplet of water) and of the probe. The whole system is connected to a controller PC running RTAI/Linux real time OS. This system is adapted to numeric control implementation.

2) Tests and results: The experiment is performed in a clean room to limit the influence of environmental conditions. The system is tested on a complex case for microscale force sensing, which consists in measuring the time-course interaction of a thin glass probe with a water droplet under direct human control. The task is split in four main phases, namely 'approach', 'contact', 'retract', and 'contact break'.

A droplet with a diameter of $0.8 \mathrm{~mm}$ is pipetted on the tip of a needle. The droplet is approached towards the probe using the position of the interface handle as a set point for the sample holder. The droplet is contacted and then retracted in approach-retract cycle. The interaction force is measured by the sensor and fed to the user through the haptic interface. Figures 9 and 10 represent respectively the force measured by the sensor over a cycle of approach-retract and the force transmitted to the operator using a probe with a diameter of $140 \mu \mathrm{m}$. The resulting curves highlight the high level of transparency of the system since the output force of the haptic interface is a linear function of the measured interaction.

The system remains stable over the experiments and achieves perfect transparency. In fact, the force felt by the user through the interface handle is exactly the force measured by the sensor amplified by $\alpha_{f}$. Hence, this investigation shows the performances and the importance of the micro teleoperation system applications. The system has also been tested by several users with no experience in microscale phenomena. Various interaction forces, like pull-in and pulloff forces, are correctly rendered to the operators as shown above.

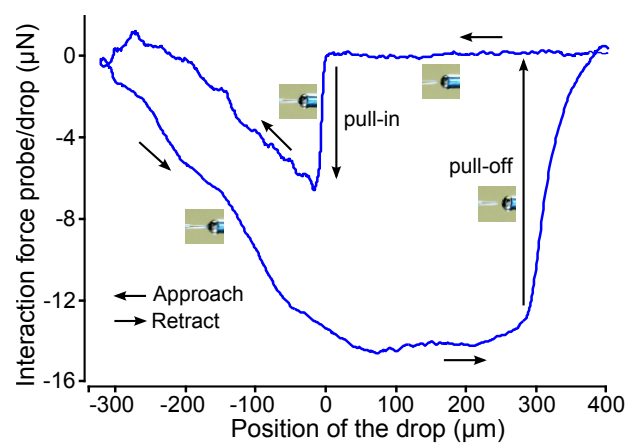

Fig. 9. Interaction force measured by the sensor over a cycle of approachretract vs the relative position between the sample and the probe.

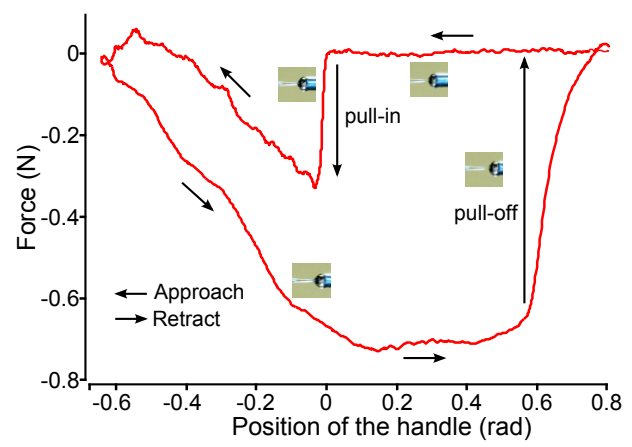

Fig. 10. Haptic force felt by the operator through the haptic vs the position of the handle.

\section{CONCLUSION}

The design of a new micro teleoperation system that is not subjected to the stability/transparency trade-off is presented. It is based on the coupling of an active force sensor with a high-fidelity haptic device through a direct bilateral control scheme. The system provides a natural and intuitive interac- 
tion with the microscale, owing to the particularities of each device. First, the force sensor provides a force measurement which is not based on a structural stiffness but on a force balancing principle. Hence its output accurately reflects the physical interaction, independently of the probe's mechanical properties. The haptic device is based on a dual stage design for a single degree of freedom. Its performances are very close to human haptic perception range. The direct bilateral coupling allows for linear transformations between microand macroscales. As a result, the system transposes successfully the sense of touch into the microscale. The system is validated by measuring the interaction of a glass probe with a droplet of water involving adhesion and capillary forces. This complex interaction is accurately rendered to the operator with unprecedented haptic clarity.

Future work would improve fabrication of the sensor using MEMS fabrication technique, while retaining the same principle. The bandwidth of the sensor is then expected to reach the $\mathrm{kHz}$ range. Another improvement would transform the rotation degree freedom of the haptic interface to translation freedom, in order to improve the system ergonomic.

\section{ACKNOWLEDGEMENTS}

This work was supported by the French Agence Nationale de la Recherche, through the NANOROBUST project, under Contract ANR 2011 NANO 006.

\section{REFERENCES}

[1] S. Régnier and N. Chaillet, Microrobotics for Micromanipulation. Wiley-ISTE, publisher, 2010.

[2] R. Hollis, S. Salcudean, and D. Abraham, "Toward a tele-nanorobotic manipulation system with atomic scale force feedback and motion resolution," in IEEE Micro Electro Mechanical Systems, 1990. Proceedings, An Investigation of Micro Structures, Sensors, Actuators, Machines and Robots, Feb. 1990, pp. 115-119.

[3] Y. Hatamura and H. Morishita, "Direct coupling system between nanometer world and human world," in IEEE Micro Electro Mechanical Systems, 1990. Proceedings, An Investigation of Micro Structures, Sensors, Actuators, Machines and Robots, Feb. 1990, pp. 203-208.

[4] D. A. Lawrence, "Stability and transparency in bilateral teleoperation," IEEE Trans. Robot. Autom., vol. 9, no. 5, pp. 624-637, 1993.

[5] G. J. Raju, G. C. Verghese, and T. B. Sheridan, "Design issues in 2port network models of bilateral remote teleoperation," in Proc. IEEE Int. Conf. Robotics Automat., pp. 1317-1321, 1989.

[6] M. Sitti and H. Hashimoto, "Tele-nanorobotics using atomic force microscope," in IEEE/RSJ International Conference on Intelligent Robots and Systems, Proceedings, vol. 3, Oct. 1998, pp. 1739-1746.

[7] J. H. Ryu, D. S. Kwon, and G. Hannaford, "Stable teleoperation with time-domain passivity control," IEEE transactions on robotics and automation, vol. 20, no. 2, pp. 365-373, 2004.

[8] M. Boukhnifer and A. Ferreira, "Passivity based control for microteleoperation system," IEEE international conference on robotics and biomimetics, pp. 416-421, 2005.

[9] R. J. Anderson and M. W. Spong, "Bilateral control of teleoperation with time delay," IEEE Transactions on automatic control, vol. 34, no. 5, pp. 494-501, 1989.

[10] G. Venture, S. Haliyo, A. Micaelli, and S. Régnier, "Force-feedback coupling for micro-handling applications," International Journal of Micromechatronics, special issue on Micro-handling, vol. 3, no. 3-4, pp. 307-327, 2006.

[11] A. Bolopion and S. Régnier, "A review of haptic feedback teleoperation systems for micromanipulation and microassembly," IEEE Transactions on Automation Science and Engineering, vol. 10, no. 3, pp. 496502,2013
[12] S. Sakaino, T. Sato, and K. Ohnishi, "Multi-DOF micro-macro bilateral controller using oblique coordinate control," IEEE Transactions on Industrial Informatics, vol. 7, no. 3, pp. 446-454, Aug. 2011.

[13] M. Mehrtash, N. Tsuda, and M. Khamesee, "Bilateral micro-macro teleoperation using magnetic levitation," IEEE/ASME Transactions on Mechatronics, vol. 16, no. 3, pp. 459-469, Jun. 2011.

[14] A. Nigues, N. Venant, M. Rodrigues, F. Comin, J.-L. Florens, and F. Marchi, "Haptic localization and shape recognition of nano objects," in IEEE/RSJ International Conference on Intelligent Robots and Systems (IROS), Oct. 2012, pp. 2319-2324.

[15] A. Schmid, R. Yechangunja, S. Thalhammer, and M. Srinivasan, "Human-operated 3D micro-manipulator with haptic feedback," in IEEE Haptics Symposium (HAPTICS), Mar. 2012, pp. 517-522.

[16] H. V. Quang and J. H. Ryu, "Implementation of time domain passivity approach on rate-mode bilateral teleoperation," 8th IEEE international conference on automation science end engineering, pp. 846-850, 2012.

[17] B. Willaert, D. Reynaerts, H. V. Brussel, and E. B. V. Pooeten, "Bilateral teleoperation ; quantifying the requirements for and restrictions of ideal transparency," IEEE transactions on control systems technology, 2013.

[18] Z. Ni, A. Bolopion, J. Agnus, R. Benosman, and S. Régnier, "Asynchronous event-based visual shape tracking for stable haptic feedback in microrobotics," IEEE Transactions on Robotics (T-RO), vol. 28, no. 5, pp. 1081-1089, 2012.

[19] A. Bolopion, B. Cagneau, S. Haliyo, and S. Régnier, "Analysis of stability and transparency for nanoscale force feedback in bilateral coupling," Journal of Micro - Nano Mechatronics, vol. 4, pp. 145$158,2009$.

[20] F. H. Peter and W. S. Mark, "Bilateral teleoperation : An historical survey," Automatica, vol. 42, pp. 2035-2057, 2006.

[21] G. Millet, S. Haliyo, S. Régnier, and V. Hayward, "The ultimate haptic device: First step," in Proceedings of the Third Joint Eurohaptics Conference and Symposium on Haptic Interfaces for Virtual Environment and Teleoperator Systems. World Haptics, 2009, pp. 273-278.

[22] A. Mohand-Ousaid, G. Millet, S. Régnier, S. Haliyo, and V. Hayward, "Haptic interface transparency achieved through viscous coupling," International Journal of Robotics research, vol. 31, no. 3, pp. 319-329, 2011.

[23] A. Mohand-Ousaid, S. Haliyo, S. Régnier, and V. Hayward, "Microforce sensor by active control of a comb-drive," IEEE/ASME International conference on Advanced Intelligent Mechatronics, pp. 612-617, 2013.

[24] J. Zhao and D. J. Hill, "A notion of passivity for switched systems with state-dependent switching," Journal of control theory and applications, vol. 1, pp. 70-75, 2006.

[25] J. C. Willems, Dissipative dynamical systems part I : General theory, S. Berlin, Ed. Archive for Rational Mechanics and Analysis, Vol. 45, N 5, pp. 321-351, 1972.

[26] D. J. Hill and P. J. Moylan, "Stability results for nonlinear feedback systems," Automatica, vol. 13, pp. 377-382, 1977.

[27] V. M. Popov, "Hiperstabilitatea sistemilor automate," Academiei, Bucharest, Romania, 1966

[28] G. Calcev, R. Gorez, and M. D. Neyer, "Passivity approach to fussy control systems," Automatica, vol. 34, no. 3, pp. 339-344, 1998.

[29] D. G. Gudrun, "Kinaesthetic feedback and enhanced sensitivity in robotic endoscopic telesurgery," Ph.D. dissertation, Leven University, Belgium, February 2005.

[30] K. F. Riley, Mathematical methods for the physical sciences. Cambridge University Press, 1974.

[31] B. Brogliato, R. Lozano, B. Maschke, and O. Egeland, Dissipative systems analysis and control: Theory and applications. Springer, 2007.

[32] J. A. Richard and B. Hannaford, "Stable haptic interaction with virtual environment," IEEE Transactions on Robotics and Automation, vol. 15, no. 3, pp. 465-474, 1999

[33] A. Bolopion, B. Cagneau, D. Haliyo, and S. Régnier, "Tuning the gains of haptic couplings to improve force feedback stability in nanorobotics," in IEEE International Conference on Robotics and Automation. ICRA '09, May 2009, pp. 4320-4325.

[34] J. E. Colgate, "Robust impedance shaping telemanipulation," IEEE Transactions on Robotics and Automation, vol. 9, no. 4, pp. 374-384, 1993. 